Témoigner Témoigner. Entre histoire et mémoire

Getuigen Revue pluridisciplinaire de la Fondation Auschwitz

$123 \mid 2016$

Traduire le témoignage

\title{
Donner la parole au bourreau
}

Scénographie et traduction dans Une saison de machettes de Jean Hatzfeld

Voicing the Perpetrator's Perspective: Translation and Mediation in Jean Hatzfeld's Une Saison de machettes

\section{Anneleen Spiessens}

\section{(2) OpenEdition}

\section{Journals}

Édition électronique

URL : https://journals.openedition.org/temoigner/5215

DOI : 10.4000/temoigner.5215

ISSN : 2506-6390

Éditeur :

Éditions du Centre d'études et de documentation Mémoire d'Auschwitz, Éditions Kimé

Édition imprimée

Date de publication : 1 octobre 2016

Pagination : 80-95

ISBN : 987 2-9600926-4-6

ISSN : 2031-4183

Référence électronique

Anneleen Spiessens, « Donner la parole au bourreau », Témoigner. Entre histoire et mémoire [En ligne],

123 | 2016, mis en ligne le 02 novembre 2021, consulté le 04 novembre 2021. URL : http:// journals.openedition.org/temoigner/5215 ; DOI : https://doi.org/10.4000/temoigner.5215 


\section{Donner la parole au bourreau Scénographie et traduction dans \\ Une saison de machettes de Jean Hatzfeld}

\section{LE BOURREAU TÉMOIGNE} $\rightarrow$ Anneleen Spiessens
Université de Gand
En premier lieu, le témoin est quelqu’un qui a observé de près et qui met en réci une expérience personnelle et catastrophique. La certification du récit s'appuie ainsi sur l'attestation biographique du narrateur qui «y était». On retient encore de la définition de Riffaterre l'association du niveau individuel au collectif: l'expérience personnelle doit cautionner une thèse plus générale sur l'histoire - une «interprétation» - et constituer ainsi une «leçon » pour le public. Alors qu'il est l'expression d'une expérience singulière vécue de l'intérieur, le témoignage fonctionne comme un monument selon Renaud Dulong (1998), incorporant une dimension mémorielle et ébauchant une tâche collective pour la société qui l'accueille. D’autres insistent sur a dimension éthiquedutémo a dimension éthique du temoignage qui se trouve intimement liee a la personne du témoignant «incarnant» son récit et évoquant, par sa présence même, le drame qui a «bouleversé» sa vie. Jacques Derrida théorise ce phénomène à partir de la question de la «signature», rappelant que le témoignage ne se réduit pas «aux rapports descriptifs, informatifs, au savoir ou au récit; c'est d'abord un acte présent» (Derrida, 1996, p. 29). Le témoignage littéraire est la représentation de cet acte.

Cette définition semble exclure $a$ priori la position de témoin au bourreau. Contrairement à la victime-témoin, on imagine mal une «leçon» cachée dans le récit personnel du tueur. Catherine Coquio renvoie ici à la différence que fait Giorgio Agamben entre testis (la personne tiers garant entre deux parties dans un procès judiciaire) et superstes (celui qui a vécu un événement dramatique et incorne la judiciaic) et superstes (celui qu a vécu un évén a des deux catégories. Incapable de témoigner de son propre crime en qualité de testis, on lui refuse aussi le statut de témoin éthique. Coquio rappelle à ce propos le témoignage d'Adolf Eichmann, indiquant que cet homme a simplement, et sans état d'âme, détaillé les circonstances de son « travail éprouvant effectué par conscience professionnelle» (2003,p. 29), sans imaginer les événements de l'intérieur et donc sans assumer la fonction de superstes. C'est encore l'intuition de Claude Lanzmann qui, interrogé par des étudiants américains, se demande s'il existe une telle chose que l' «expérience des bourreaux» (Lanzmann, 1995, p. 212).

Malgré ces réserves, la littérature peut rendre compte du récit du bourreau. Pas «au premier degré» (Milkovitch-Rioux, 2010, p. 193), toutefois, mais par l'élaboration d'une mise en scène particulière où le récit du tueur est «médié» ou «traduit» - voiced, dirait-on en anglais - par une autre instance narrative. Au lieu de refuser donc d’accorder à cette narration le statut de témoignage, nous suggérons qu’elle négocie plutôt sa place et sera le résultat d’un travail discursif poussé.

LES INSTANCES DISCURSIVES DANS UNE SAISON DE MACHETTES

Ayant visitéle Rwanda en qualité de journaliste pendant l'été 1994, Ayant visitéle Rwanda en qualité de journaliste pendant lété 1994 ,
Jean Hatzfeld y retourne quelques années plus tard pour monter un
- Alfredo Jaar « Rwanda 40 enseignes publicitaires a Malmo (Suède) pour The

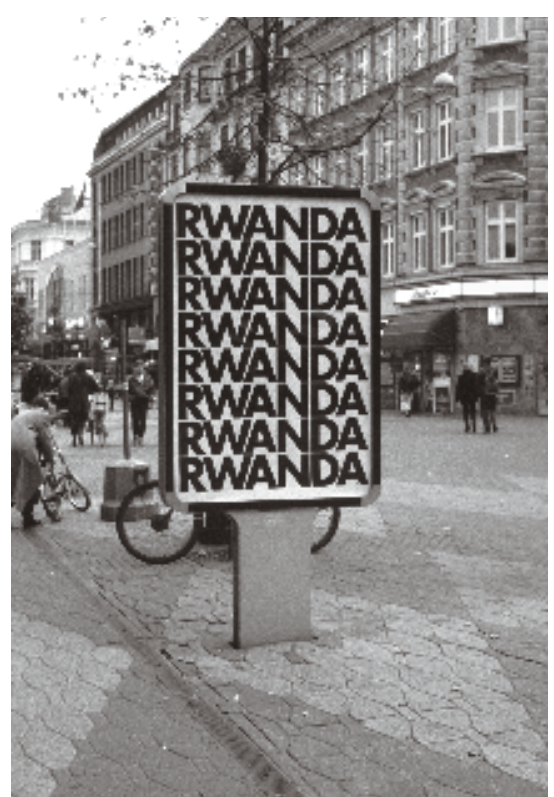


projet très personnel. Destination : la commune de Nyamata dans le Bugesera, à 30 kilomètres au sud de Kigali. En un seul mois, 50000 Tutsis y ont été massacrés sur une population de 59000. De nombreuses personnes sont mortes dans les église de Nyamata et de N'tarama, transformées aujourd'hui en sites mémoriaux. Après avoir écouté les victimes pour son premier livre Dans le nu de la vie (2000, Prix France Culture), Hatzfeld entre en dialogue avec une bande de tueurs originaires de la même région et emprisonnés dans le pénitencier de Rilima. Les conversation constituentle point de départ d'Une saison de machettes (2003 Prix Femina Esaiet constitue Prix Joseph Kessel) ${ }^{2}$.Quand en 2003 le gouvernement rwandais applique sa politique de réconciliation et libère 40000 genocidaires de ses prisons surpeuplées, Hatzfel retrouve pour La Stratégie des antilopes (2007, Prix Médicis) les personnages de deux livres précédents, forcés aujourd'hui de cohabiter sur les collines de Nyamata. Les trois livres proposent une transcription de témoignages oraux délivrés à la première personne, alternant avec des commentaires et des impressions de l'auteur.

Si sa première visite au pénitencier de Rilima date déjà de son séjour pour Dans le nu de la vie, Hatzfeld attend plusieurs années avant de reprendre le fil avec les génocidaires et d’intensifier le contact curieux finalement de savoir «ce qui s’était passé dans la tête des tueurs." (MA, p.49) De façon confuse, estime Destime l'auteur, les tueurs veulent souligne devant exterminater, une angoissante vérte.» (MA, p. 270) Mônne si cette vérité ne sera pas incompatible avec Yinvention, linagination et le mensonge, Hatzfeld es convaincu que les récits « reflètent une vérité plus essentielle» sur l'atmosphère et la mentalité des hommes au moment des massacres. Chaque étape du projet se double toutefois d'une interrogation consciencieuse sur la légitimité et les conditions de possibilité de l'entreprise, signe de l'embarras de l'auteur : « est-il moral, non pas de parler avec de tels tueurs, mais de les encourager à s'exprimer?» (MA p. 263), se demande-t-il.

Dans la suite, nous nous interrogerons sur le statut du « témoignage» des génocidaires rwandais dans Une saison de machettes. Loin de considérer leur parole comme une donnée statique, nous en soulignerons la nature construite. L'on ne saurait, une donnée statique, nous en soulignerons la nature construite. Lon ne saurait, en effet, sous-estimer le travail d'écriture qui est à la base du livre et qui diffère du
procédé choisi pour Dans le nu de la vie. La position de l'auteur tiers change bien sûr procédé choisi pour Dans le nu de la vie. La position de l'auteur tiers change bien sûr côté» ainsi que les doutes sur la légitimité de sa démarche aboutissent à une prise de position forte de la part de l'auteur, intervenant comme un narrateur omniscient et dirigeant les entretiens avec rigidité. Hatzfeld n’assume pas d'emblée son propre travail de mise en scène, opérant d'après Coquio un brouillage «camouflé, mais volontaire» (2004, p. 170) de plusieursétapes d'écriture Les propos des tueurs sont en fat soumis à un pro) de de transcription, de rér et de traduction dont toute trace a été effacée dans le texte, mais dont on ne peut
minimiser les effets.
Au lieu de considérer les propos dans Une saison de machettes comme un témoignage «brut» laissant entendre la «voix» des bourreaux, l'on s'engage à étudier précisément la nature construite de cette parole et à regarder de plus près le rôle que joue Hatzfeld. L’analyse du livre remet en cause le modèle selon lequel l'on pourrait ramener chaque discours à une source énonciative unique - à une voix qui serait à elle seule responsable de la production du texte. Hatzfeld, lui, fait pourtant croire que son ouvrage se conforme à un schéma discursif dont la simplicité apparente est probablement trompeuse :

(text)

[I say : (text)]

\{I translate: : [I say: (text)]\} (Hermans, 2010, p. 209)

On peut repérer ici deux niveaux d'énonciation, deux «voix» ou deux instances discursives. Le «texte» ou témoignage est émis par le bourreau (le premier «Je» dans le schéma) pour ensuite être repris - cité ou renarré - par Hatzfeld en tant quauteur-narrateur. Hatzfeld fera tout pour maintenir l'illusion de produire un discours rapporté où le rapporteur ne fait que répéter, à partir d'un « dehors » minutieusement construit, les mots des tueurs. Selon ce raisonnement, le modèle des traductions néerlandaise et anglaise du livre ressemblerait à ceci :

(text)

[I say : (text)]

$\{$ I re-narrate : [I say : (text)]\}

$<$ I translate : \{I re-narrate : [I say : (text)]\} $>$

On ajoute un troisième niveau d'énonciation : celui du traducteur qui, selon une conception naïve de la traduction, reflète « fidèlement » le discours de Hatzfeld, qui cite à son tour les tueurs.

Le modèle ci-dessus est intenable pour deux raisons. Premièrement, Hatzfeld passe largement sous silence le travail de traduction et d'interprétation (du kinyarwanda vers le français rwandais) qui précède sa propre activité de «citateur ». La présence del'interprète/traducteur, même si elle a été gommée des entretiens, doit être introduite dans le schéma narratif pour pouvoir en étudier le rôle et l'efficacité:

(text)

[I say: (text)]

$\langle$ I translate : [I say : (text)] $\rangle$

$\{$ I re-narrate :\{I translate: [I say : (text)] $\}\}$

$<$ I translate: $\{$ I re-narrate $\langle$ I translate : [I say : (text) $]\}\}>$

Ce modèle ne nous semble toujours pas adéquat. Même s'il ajoute un niveau discursif qui reflète mieux la polyphonie du texte, il suggère aussi une stricte séparation 
des voix etévoque la possibilitéd'un discours original qui ne soit en rien affectépar les mises en forme successives par l'auteur et les traducteurs. Notre analyse démontrera que les voix s'entremêlent forcément, chaque niveau discursif provoquant des glissements et des déplacements, voulus ou non, dans le discours du bourreau. Ni le travail de Hatzfeld qui recueille les témoignages ni l'activité des traducteurs ne peuvent être considérés comme de pures «citations» relevant du discours indirect. L'auteurnarrateur et les traducteurs sont manifestement co-producteurs du témoignage des génocidaires, et de leur intervention feront l'objet d'une analyse détaillée.

LA SCÉNOGRAPHIE DU TÉMOIGNAGE

L'ouvrage entier ension où Hatzfeld l'am exploite l'ambiguïté générique créée par 'imprécision du soustitre «récits», un terme à cheval entre fiction et non-fiction qui prête à confusion On sait que la trilogie sur le Rwanda est cen-

- «The Silence of 1 million de diapositives, table lumineuse et loupe pour The Rwanda Project,
1994-2000. sée corriger l'échec journalistique de 1994 et marquer, pour Hatzfeld, son entrée sur

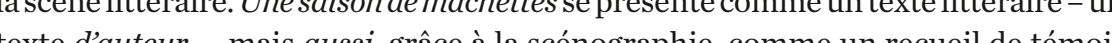
texte dauteur-, gnages «bruts » et authentiques qui prétend faire entendre la «voix» du génocidaire Contrairement au premier livre caractérisé par une nette orientation littéraire, c'est l'aspect documentaire qui l'emporte dans Une saison de machettes. Cette évolution est sans doute liée au projet particulier et moralement ambigu d'aller parler avec des bourreaux dont on sait la combinaison avec l'écriture littéraire peu évidente. Assurément, bien que Hatzfeld publie aux Éditions du Seuil, qui plus est dans la collection «Fiction \& Cie», il serait erroné de qualifier les «récits» de «fictions». Jean-Pierre Karegeye suggère néanmoins que les transformations que fait subir l'auteur aux témoignages apportent «une teinte fictionnelle aux récits factuels (2010, p. 218). On se demande en effet si Hatzfeld ne fait pas exprès de balancer son texte sur la fine ligne entre reportage et littérature pour occulter son travail de réécriture.
La qualité documentaire de l’ouvrage et l'authenticité des narrations sont mises en avant par le paratexte d'abord, où le lecteur retrouve une chronologie des événements, un glossaire expliquant des éléments de la culture rwandaise, des cartes du pays et une biographie des tueurs - y compris leur jugement - accompagnée d'une photo de groupe. La traduction anglaise, sous-titrée A report by Jean Hatzfeld, insiste davantage sur la valeur de ce que l'éditeur considère être un document historique. "A trove for future historians and ethnographers seeking to explain the mechanism of genocide», lit-on sur la quatrième de couverture de of genocide», lit-on sur la quatrieme de couverture de 'edition américaine. Citant OMagazine, l'editeur améicain ajoute que le lecteur peut parfaitement entendre «the voices of the men, many of whom speak in a kind of chilling, breathtaking poetry.» Parallèlement, lédition britannique présente le livre comme un «chilling reportage» qui «documente» si bien «the voices of Rwandan killers». Selon Ron Dudai, qui fait le compte rendu, le livre est choquant parce que les témoignages des bourreaux sont «painfully unmediated» $(2006$, p. 700$)$.

Hatzfeld maintient l'illusion d'une voix criminelle pure et directe en faisant alterner, dans des chapitres pure et directe faistres séparés, la parole des tuenrs et celle de lauteur. La séparation rigoureuse des chapitres cree un «dehors » auctorial qui permet à l'auteur de juger et de commenter ouvertement ses interlocuteurs sans que sa propre «voix» ne se mêle à celle des tueurs. De cette façon, Hatzfeld crée pour lui-même un lieu discursif d'où il peut formuler un contre-discours qui dévoile sa propre position vis-à-vis du discours de ses interlocuteurs.

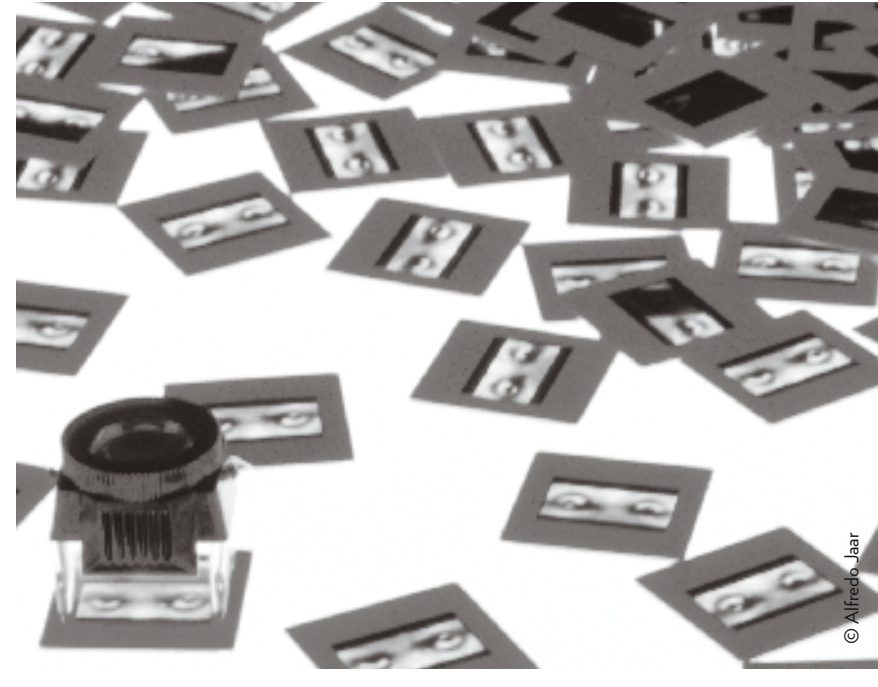

-The Silence of Nduwayezu
(close-up).
Le cadrage n'est pas seulement visuellement présent dans le livre ; la distance entre l'auteur et ses interlocuteurs est davantage mise en lumière par l'emploi du lanentre l'auteur gage, et plus particulièrement par la présence de deux variantes de français. Laalternance entre le «français rwandais» des témoins, «parlé» et hautement imagé, et le «français de l'Hexagone», parfois même poétique, de l'auteur structure la narration dans l’ouvrage. La scénographie double crée deux discours séparés auxquels le lecteur associe deux «tons » ou « voix » différents qui désigneraient l'origine énonciative. Il est évident que, en créant ce langage «typiquement rwandais» et inséparable des atrocités qu'il doit révéler, Hatzfeld contribue aussi à renforcer les clichés existants sur le rapport entre la violence «primitive» et l'identite culturelle africaine.

Nous nous proposons d'étudier la scénographie que propose l'auteur-narrateur dans Une saison de machettes et les stratégies discursives qu'il élabore pour légitimer son œuvre au niveau épistémologique (l'authenticité du témoignage) et moral 

de l'auteur de s'éloigner des génocidaires, la mise en scène, attribuant à chaque discours sa vocalité propre, manque de transparence et escamote la complexité de la construction textuelle.

\section{LE DISCOURS AUCTORIAL}

Arrêtons-nous d'abord sur la composition et la fonction des chapitres réservés à l'auteur-narrateur qui relient les entretiens entre eux et les placent dans leur contexte socio-historique. Paul Kerstens fait remarquer que, contrairement au premier livre où ces chapitres étaient plus concis et se limitaient à une présentation des témoins dans leur environnement naturel, Hatzfeld s'érige dans Une saison de machettes en «narrateur omniscient » (Kerstens, 2006, p. 102). Il réfléchit amplement sur l'histoire du Rwanda et ses structures sociopolitiques, se plonge dans des méditations philosophiques sur le pardon (avec des références à Ricœur, MA, p. 219), commente le comportement des tueurs et s'essaie à une psychologie du bourreau quand il découvre qu'ils ne souffrent pas de troubles psychiques ni même de cauchemars: «À quoi doivent-ils la mansuétude de leur inconscient leurbizarre faculté à barrer la porte du dortoir à leur culpabilité ? (MA, p. 225) Les chapitres centes ustrée dans les entretiens avec les tueurs.

Le discours auctorial, toutefois, ne sert pas uniquement à fournir un contexte aux entretiens; il propose aussi un métadiscours sur les conditions de possibilité et la nature de la parole criminelle. Le projet de Hatzfeld consiste ainsi en une quête de la parole sincère, de la réponse à la question du «pourquoi» qui ne relève pas de l'apologie ou de l'imposture. En dépit d'un contrat formel avec ses interlocuteurs et d’un travail de sélection rigoureux, l'auteur n’a pu éviter que les récits contiennen des mensonges «un ou deuxqueje connais, d’autres pas encore.» (MA p. 157) Même après avoir exclu les imposteurs les plus persévérants du groupe, l'auteur ne cache apres ache

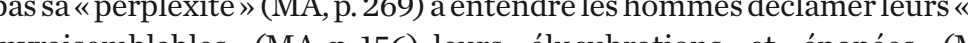
invraisemblables» (MA, p. 156), leurs «élucubrations» et «épopées» (MA, p. 265)

À première vue donc, la scénographie «bipolaire» procure au livre une structure narrative transparente qui permet à l'auteur d'exposer la fausseté de certain récits et d'afficher sa position morale sans pour autant compromettre l'idée d'un témoignage authentique. A y regarder de plus près, la frontière séparant le discours auctorial de celui des tueurs présente des fissures par où d'autres voix s'insinuent dans les témoignages. C'est d'abord la voix de l'auteur-narrateur qui s'infiltre dans le témoignage des génocidaires. En effet l'écriture même du livre repose sur plu-

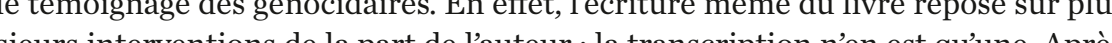
sieurs inter avoir éte transcits, les la part de laux témoignages restants sont ensuite regroupés en neuf chapitres thématiques traitant d'un aspect particulier du génocide et pourvus de titres tendancieux qui provoquent des images rhétoriques et orientent fortement l'interprétation des récits: «Le goût et le dégoût » suggère que les hommes prennent plaisir à tuer ; dans «La fête au village» Hatzfeld mentionne les pillages qui accompagnent les massacres et qui doivent illustrer l'absence de morale chez ses interlocuteurs ; les tueurs comparent le coupage de légumes au «coupage» de personnes dans «Travaux des champs» ; et

À l'intérieur des chapitres testimoniaux, ensuite, les paroles des tueurs sont rapportées sous forme de monologue ininterrompu. La présence de l'auteur lors des interviews a été parfaitement gommée, de sorte qưil est impossible de repérer les questions qu'il a posées et donc de connaître son influence sur le vocabulaire des tueurs. La fiction du monologue est une stratégie narrative puissante, puisqu'elle suggère en outre une certaine facilité de la parole. L'apparente aisance avec laquelle les tueurs s'expriment influence naturellement leur ethos et suggère l'absence, chez eux, d'une conscience morale. Sans être véritablement perturbés, ils parlent de leur premier meurtre, de la distraction de la «chasse», du travail éreintant dans les marais et de leur espoir d'être pardonnés. Hatzfeld revient à plusieurs reprises es mares sur la fanneuse «placidtém de ses interlocuteurs, qualiffantleurvoix de «régulière» ou de «monocorde» (MA, p. 266, p. 172). Quoiqu il soit indéniable que la parole du bourreau frappe par sa précision et sa legèreté, faisant contraste avec la gravité des enjeux, l'on ne saurait minimiser l'effet de la stratégie d'écriture de l'auteur, qui est coresponsable de la construction de cette « voix» et aide à faire apparaitre les tueurs comme des personnes monstrueuses.

\section{LE RÔLE DE LINTTERPRÈTE}

Jean Hatzfeld témoigne d'une confiance inébranlable en la traduction, qui étaie ses ouvrages d'une manière plus structurelle qu'il n'y paraît à première vue. Dans le vue. Dans le premier to solant que «ces recits sexprinent en trois langus : le kinyarwanda, langue des cultivatrices; le français rwandais, langue des autres personnes et des traducteurs; et le français de l'Hexagone» (Hatzfeld, 2000, p. 13). La Stratégie des antilopes révèle ensuite quelques caractéristiques du kinyarwanda : c'est une langue «concise, d'une grammaire incroyablement sophistiquée, comprise seulement des Burundais, et imperméable au swahili, au français ou à l'anglais.» (Hatzfeld, 2007, p. 89) Le français rwandais a des qualités poétiques dues à l’appropriation du vocabulaire français jugée «magnifique» par Hatzfeld (Hatzfeld, 2000, p. 13).

À partir de ces indications, on peut conclure que le projet testimonial, et particulièrement Une saison de machettes, dépend largement de la traduction et de ticulièrement Une saison de machettes, dépend largement de la traduction et de
l'interprétation. Si le kinyarwanda est la langue des cultivateurs comme l'affirme 
Hatzfeld dans Le nu de la vie, elle a dû être la langue véhiculaire pour les entretien avec les Hutus, sélectionnés à dessein parmi la population cultivatrice. Hatzfeld a jeté son dévolu sur ces hommes, explique-t-il, pour plusieurs raisons : ils sont liés par l'amitié, ils habitent les mêmes collines que les rescapés du premier livre, et ils sont «cultivateurs, sauf un fonctionnaire et un instituteur » (MA, p. 50) - c'est-à-dire qu'ils n’appartiennent pas à des milices, mais représentent idéalement le caractère «populaire» de ce génocide «de proximité» (MA, p. 74).

Autre critère pour la sélection des témoins : leur relation à Innocent Rwililiza, un professeur de lecole locale qui prend maintenant le rôle d'interprète pendant les conversations. «Il est l'intermédiaire indispensable, plus le collaborateur idéal, et le traducteur formidable quand cela s'avère nécessaire», souligne Hatzfeld (MA p. 51). Le lecteur apprend qu'Innocent a également fourni les traductions pour Dan le nu de la vie et qu'il a partagé le travail avec Sylvie Umubyeyi, assistante sociale et rescapée comme lui. Leur méthode de travail était identique à celle d'Innocent avec les prisonniers :

La mise en marche du magnétophone signale le début de l'entretien, en français, ou en kinyarwanda traduit par Innocent.

À ce propos, des lecteurs du précédent livre ont évoqué l'influence du traducteur sur des rescos sylie Sylvie et Innocent, les deux interprètes, avaient compris qưune retranscription des textes in extenso était la première étape nécessaire pour passer de l'oral à l'oral écrit. Ils avaient donc si finement et fidèlement traduit qu'i est impossible, même à un Rwandais francophone, de distinguer les récits traduits et ceux livrés directement en français par le rescapé. Il en est de même pour ces récits, même si le mode d'expression des tueurs est aussi différent que leur motivation à s'exprime (MA, p. 169-170)

Il découle de ces précisions que tant l'enquête que l'écriture d'Une saison de machettes reposent dans une très large mesure sur l'intervention d'Innocent. Il inquiétant, dès lors, de constater que Hatzfeld expose dans ces lignes une concepinquiétant, des lons, de con tion éminemment haive de la traduction. Les traducteurs du prenier live, comme Innocent pour le denxiène, auraient impossible de distinguer les récits traduits et ceux livrés directement en français par le témoin. Autrement dit, une illusion efficace a été obtenue qui a été dénoncée pa Venuti : on ne s'aperçoit pas de la différence entre le langage traduit et l'original c'est-à-dire que la traduction ne ressemble pas à une traduction :

Under the regime of fluent translating, the translator works to make his or her work «invisible », producing the illusory effect of transparency that simutaneously masks its statu

Le texte est fluide et transparent, la traduction «naturelle» puisque invisible en tant que traduction, même pour un Rwandais francophone. Nulle part, il n'est précisé quels discours ont été traduits et lesquels ont été fournis directement en français rwandais. Ce problème est bien sûr aggravé par le travail de compilation des témoignages, dispersés sur plusieurs chapitres thématiques. La voix d’Innocent n'est par conséquent pas audible et la remarque métadiscursive de Hatzfeld mise à part, le traducteur disparaît complètement derrière sa traduction.

L'illusion de la non-traduction est brisée par une analyse détaillée du contexte et des circonstances des entretiens. Rien ne contredit le fait qu'Innocent soit effecet descircon tivement un «formidable» traducteur du kinyarwanda - bien qu'on se demande si Hatzfeld est bon juge en la matiere-, mais on ne peut soutenir que son intervention soit anodine (innocente?) etn'nfuencentien la narrationdes tueurs. Ainsi on peut s'interroger sur le choix des mots des Hutus, abondamment commenté et qualifié d'évasif par Hatzfeld. Là encore, il serait malséant de prétendre le contraire, mais l'on se rend compte que les formulations des tueurs - certaines assez surprenantes comme chez Fulgence («une optique finale», MA, p. 251) ou Pancrace (tuer «jusquau dernier des derniers», MA, p. 251, 252) - sont en réalité, en plus d'être la réponse à une question inconnue de Hatzfeld, des traductions d'Innocent.

L’auteur ne manque pas de préciser, en outre, le rapport très personnel entre son interprète et les tueurs. Il cite Innocent lui-même qui affirme connaître les garçons «depuis longtemps auparavant» et même avoir enseigné à quelques-uns (M, p. 51), «depuis longtemps auparavant» ainsi dans une position d'autorité. Dans le premier tome de la trilogie, le plaçant ainsi dans une position d’autorité. Dans le premier tome de la trilogie, nous lisons en outre son histoire personnelle: son épouse et son enfant ont été tués la bande et inversement :

Léopord, lui, il ne se faisait remarquer de rien sauf de sa longueur. Sauf aussi que ce qưil a fait de sa machette, par après, est extraordinaire. Ignace était aussi usé que rusé, de plus il se montrait soudainement méchant en face de Tutsis (M, p. 51).

Ces informations aident manifestement Hatzfeld à évaluer les narrations des tueurs, à interpréter leurs hésitations et à repérer les mensonges. Pour le lecteur aussi, cette présentation est utile pour s'orienter dans les témoignages assemblés. Cela n'empêche qu'elle influence fortement l'image des prisonniers que se forge le lecteur, les transformant en des personnages de livre et les jugeant avant même qu'ils ne prennent la parole.

\section{TRADUIRE UNE SAISON DE MACHETTES}

Le travail d'interprétation (lors des entretiens) et de traduction (lors des transcriptions) de la part d'Innocent constitue en fait la mise en abyme d'une autre étape d'écriture qui accompagne la parution du livre à l'étranger. En 2005, deux 
ans après la publication française, Une saison de machettes est traduit en anglai sous le titre A Time for Machetes. The Rwandan Genocide - The Killers Speak et Machete Season. The Killers in Rwanda Speak, chaque version préfacée par Susan Sontag. Bien que le titre, la présentation de l'auteur et de son œuvre, ainsi que la couverture du livre diffèrent d'une version à l'autre, la traduction faite par Linda Coverdale est identique et a été couronnée du Scott Moncrieff Prize. La préface de Sontag est incluse dans Seizoen van de machetes. Het verhaal van de daders, dont Théo Buckinx assure la traduction ${ }^{3}$

Les traducteurs néerlandais et anglais sont les dernières « voix» intermédiaire qui peuvent altérer la configuration des différents niveaux d'énonciation et la structure «bipolaire» du récit. On a pu constater que la scénographie originale repose sur la coexistence du français rwandais et du français «de l'Hexagone» dans le même texte, et en particulier sur l'opposition entre les deux. Les variantes du français déterminent la tonalité du texte et facilitent pour le lecteur l'association d'un discours à un ethos spécifique - à une voix donc, et à un caractère. Les traducteurs recourent à un certain nombre de stratégies qui servent, dans certains cas, à maintenir cette scénographie.

D’abord, nous constatons que l'anglais tente de reprendre autant que possible le vocabulaire peu conventionnel du français rwandais et les combinaisons inusitées entre adjectifs et substantifs :

(a) Au début, [les amendes] étaient très pénalisantes pour le cultivateur à cause de sa pauvreté. (M 83)

At first these fines were very punishing for a farmer because of his poverty

(MA, p. 67)

In het begin was het een zware straf voor de boeren vanwege hun armoede.

$(\mathrm{MN}, \mathrm{p} .87)$

(b) Je ne crois pas que les vaches présentaient un détestable problème. (M, p. 256)

I do not believe the cows presented a truly hateful problem [...] (MA, p. 208)

Ik denk niet dat de koeien zo'n groot probleem vormden. (MN, p. 253)

Les amendes «pénalisantes» demeurent «punishing» en anglais (a), le « détestable problème» des vaches devient «a truly hateful problem» (b). En néerlandais, par contre, la spécificité linguistique du français rwandais ne se fait pas remarquer. Les lecteurs auront du mal à «entendre » deux voix distinctes dans le livre, puisque tant Hatzfeld que les tueurs parlent un français idiomatique. Les amendes sont «zwaar» [lourdes] au lieu de punissables (a); le «problème détestable» que soulève Alphonse dans (b) devient «een groot probleem» [un grand problème].

Le langage des tueurs est en outre riche en images générées par des personnifications ou des expressions métaphoriques à teinte exotique : (c) La chance n'exemptait aucun Tutsi dans les marais. (M, p. 134) Luck did not exempt a single Tutsi in the marshes. (MA, p. 111) In het moeras kwam geen enkele Tusi er onderuit. (MN, p. 139)

(d) Ce massacre, on n'en parlait pas avant, parce que c'était une affaire des intimidateurs qui se préparait à l'écart des oreilles. (M, p. 258)

That massacre, we did not speak of it beforehand because it was the business of the intimidators and was being prepared outside of earshot. (MA, p. 219) Tevoren werd er niet over bloedbaden gesproken, dat was een zaak van de Tevoren werd er niet over bloedbaden gesproken, dat was een zaak
aanvoerders, die zich in het geheim voorbereidden. (MN, p. 266)

(e) C'est surtout la terrible vie de la prison qui découpe mon sommeil. (M, p. 179) It's mostly this wretched prison life that cuts into my sleep. (MA, p. 149) Het is vooral het vreselijke gevangenisleven dat me uit mijn slaap houdt. (MN, p. 185)

La personnification dans (c) contribue à rendre le langage des génocidaires poétique et est reproduite en anglais. Quand Fulgence prétend ensuite que le génocide été préparé «à l'écort des orilles (d) l'angis opte pour «outside of earshotride a expression quimaintientla réche (d) émoussé par le mauvais emploi de la préposition (out of earshot est correct). Pour dire que les cauchemars le tenaillent à cause de son emprisonnement, Alphonse utilise l'expression étonnante «découper le sommeil» (e). En anglais, Linda Coverdale reproduit le style du tueur en disant «cut into my sleep». En néerlandais, on remarque, dans (c), la préférence de Théo Buckinx pour les expressions idiomatiques : dans les marais, aucun Tutsi «n’y échappait» (er kwam geen enkele Tutsi onderuit). Dans les deux autres extraits, le traducteur néerlandais opte également pour une traduction fluide et idiomatique qui efface l'effet visuel de l'original. Une préparation «à l'écart des oreilles» se fait «in het geheim» [en secret, en cachette] (d) - le malheur en prison « houd t me uit mijn slap [ [m' (e). Ce dernier exemple est d'ailleurs révélateur de la tendance de Buckinx àatténuer le vocabulaire cru des tueurs, en supprimant le verbe « couper» (hakken) pour y substituer des formules moins marquantes comme «tuer» (doden, MN, p. 141), «assassiner» (vermoorden, MN, p. 140), ou «frapper» (slagen, MN, p. 154).

Il sera clair que le texte anglais restitue le langage imagé et métaphorique des tueurs, et maintient la syntaxe spécifique - y compris les personnifications - du français rwandais. À la lumière des observations de Lawrence Venuti sur l'idéologie de «fluidité» dominant généralement la traduction anglaise (cf. Venuti, 1995, p. $5 ; 1998$, p. 4), il est surprenant de voir que Linda Coverdale adopte une stratégie à cette strágie, langlis des tueurs est grammaticalument la cette stratégie, langlais des tue de 

Donner la parole au bourreau
(suite)

lecteur peut clairement «entendre » deux voix distinctes et s’aperçoit des différences linguistiques qui forment la base de la scénographie originale de Hatzfeld.

Dans le texte néerlandais, par contre, l'auteur et les témoins parlent le même langage. L'opération de standardisation dans le discours « criminel» supprime toutefois la signification éthique des spécificités linguistiques et stylistiques du français original. Il est difficile de voir les raisons de ce choix, mais le résultat est évident: le texte devient «plato, de manière qu'il ne reste que la séparation visuelle des cha(ix chapitres pour soutenir la scenographie double de Hatzfeld. En outre, on a remarque que le traducteur atténue le vocabulaire cru et précis des tueurs. Cette intervention a des conséquences importantes : criminelle, qui parait moins froide et distante, et modifie ainsi l'image du bourreau tel que Hatzfeld le construit dans sa propre version.

Les glissements dans la traduction néerlandaise donnent lieu à une autre scénographie dont la différence avec l'original passe largement inaperçue pour le lecteur étranger - sauf dans le cas exceptionnel où il compare la traduction à la version française. Toutefois, l'illusion d'une traduction «concordante» ou «coïncidente» est brisée pour le lecteur à des moments où la voix du traducteur se manifeste explicoxplicitement d'Une sisonçaise

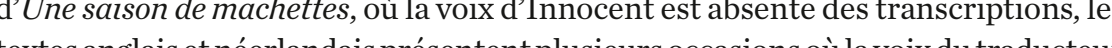
textes anglais et néerlandais présentent plusieurs occasions où la voix du traducteu apparaît ostensiblement comme instance discursive. Theo Hermans indique que cette voix surgit dans les «inégalités et les interstices » du texte et plus précisémen dans des cas de «performative self-contradiction» (Hermans, 2010, p. 209 ; p. 198) That We Didn't Know » sur trois écrans pour The
Une telle contradiction peut naître, d'abord, de l'inscription culturelle du texte. Ainsi, la traductrice anglaise est présente dans ses «notes du traducteur» qui précisent les notions de cabaret (MA, p. 3) et de paroisse (MA, p. 23) qui sont moin évidentes pour le lecteur américain ou britannique. Une intervention s'impose parfois pour expliciter un élément apparemment «intraduisible», comme la différence subtile, mais significative entre «survivant» et «rescapé» en français (MA, p. 146 MA, p. 180). Hermans cite enfin ces moments où le texte cible attire l'attention sur - ou rend visible - l'acte même de traduire dans un effort d' «autoréflexivitén ou d' d'auto remploi des mote dans le texte, acconpagnes din traduction : les bourreaux renplacent le not genocide par «"massacres", itsembatsemba, ou plus souvent "guerre", intambara» (M, p. 174) Déjà dans la version française, l'introduction du kinyarwanda crée un «credibility gap » (Hermans, 2010, p. 200) : le lecteur est confronté au processus d'interprétation qui précède la transcription des entretiens, mais dont le lecteur ne retrouve aucun trace. En traduction, ces remarques vont provoquer un court-circuit. Coverdale et Buckinx fournissent leur propre traduction des mots en kinyarwanda, traduisan ainsila traduction d'Innocent («itsembatsemba "massacres", and especially intambara "war" " MA p. 145 ; «"bloedbaden”, itsembatsemba, of vaker nog "oorlog", intambara MN p. 180). Ce procédé sous-tend évidemment le livre entier, intambara», MN, p. 180). Ce procédé sous

\section{OLYPHONIE ET POSITIONNEMENT}

Hatzfeld élabore pour son livre une scénographie très particulière qui repose essentiellement sur la séparation de son propre discours de celui des tueurs, distance qui lui permet de commenter les récits d'un «dehors » discursif sans compromettre l'authenticité du témoignage. Une telle scénographie crée toutefois l'illusion d'une nette séparation entre la voix de l'auteur et celle nette séparation entre la voix de lauteur et celle du tueur. Nous avons précisement voulu mettre en évidence la nature polyphonique du témoignage, qui n’est en rien une donnée «brute», mais est profondément affecté par le processus décriture comprenant la transcription, la sélection, le montage et la traduction des récits. Hatzfeld en effet gère mal certains aspects linguistiques de son projet, a commencer par sa confiance inébranlable dans les traductions «fidèles» d'Innocent et le manque de transparence sur le statut du kinyarwanda lors des entretiens. La présence discursive des traducteurs étrangers à des endroits où le texte se ucoucteurs étrangers a des entention sur le sujet du lart-cirde la traduction. 
otre analyse a pu révéler la complexité de la composition textuelle dans Une saison de machettes. L'entremêlement des voix dans le livre et dans ses traductions n’enlève toutefois rien à la valeur du projet de Hatzfeld. Il est vrai que nous n’avons pas accès à la «matière première » du livre - les enregistrements des entretiens ou même la transcription brute -, mais ce n'est pas, comme l'indique Claudine Vidal, ce qu’on attend d'un «écrivain » (Vidal, 2006, p. 29). L’on ne met certainement pas en question la démarche de l'auteur, quipeuten soi s'avérer parfaitement justifiée et émane sansaucun douted'uneprofon

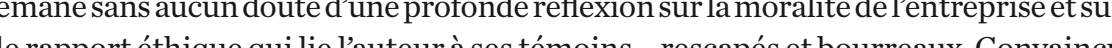
le rappoot éthiquequlielaur quilily a là un accorde pas la parole sous les mêmes conditions qu’aux rescapés.

Ce qui manque donc dans le livre, c'est une véritable autoréflexivité et une reconnaissance de la position «impliquée » de l'auteur-narrateur. Hatzfeld n'assume pa entièrement son travail de mise en scène et prétend à l'objectivité, estimant que c'est la seule manière de rendre son entreprise acceptable. Nous argumentons précisément le contraire : il n'est pas possible, et même pas souhaitable que l'auteur soit «impartial» en reprenant le discours du bourreau. Il est tout à fait légitime pour Hatzfeld de couler la distance morale qui le sépare du tueur dans une forme pour Hex textuelle. Seule en aucun cas être localisée dans un «dehor
profondément le témoignage du bourreau.

Une version élaborée de cet article est parue dans Spiessens, 2016.

\section{TEXTES PRIMAIRES}

Français

- Hatzfeld, Jean (2000) : Dans le nu de la vie. Récits des marais rwandais, Paris, Seuil.

--- (2003) : Une saison de machettes, Paris, Seuil.

-.-- (2007) : La Stratégie des antilopes, Paris, Seul.

Anglais - UK

- Hatzfeld, Jean (2005): Into the Quick of life: The Rwandan Genocide
- The Survivors Speak, traduit du français par Gerry Feehily, London, - The Survivars
Serpent's Tail

---- (2005) : A Time for Machetes. The Rwandan Genocide: The Killers Speak, traduit du français par
Sontag, London, Serpent's Tai.

- -.-- (2009): The Strategy of Antelopes: Rwanda After the Genocide,
traduit du français par Linda Coverdale, London, Serpent's Tail.

Néerlandais

- Hatzfeld, Jean (2004): Seizoen van de machetes. Het verhaal van de
daders, traduit du français par Théo Buckinx, Amsterdam, De Bezige Bij. -.-- (2008): De strategie van de antilopen. Daders en slachtoffers van de
Rwandese genocide, traduit du français par Alice Teekman, Amsterdam,
De Bezige Bij.
TEXTES SECONDAIRES

- Coquio, Catherine (2003) : « À propos d'un nihilisme contemporai : négation, déni, témoignage n, in Lhistoire
témoignage, Nantes, LAtalante, p. 23-89.

--- (2004): Rwanda. Le Réel et les récits, Paris, Belin.

- Devarrieux, Claire et Levisalles, Natalie (7 novembre 2006) * " Les Bienveillantes, roman à controverse ", Libération.

- Dudai, Ron (2006) : « Understanding perpetrators in genocides and

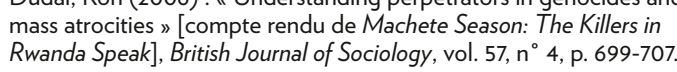
- Dulong, Renaud (1998) : Le Témoin oculaire. Les conditions sociales de
lattestation personnelle, Paris, Editions de l'EHESS. - Hermans, Theo (2010) : "The Translator's Vice in Translated
Narrative » (1996], in Mona Baker (dir.), Critical Readings in Translation

- Karegeye, Jean-Pierre (2010): « Linsscription du bourreau dans les

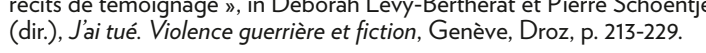
- Kerstens, Paul (2006): : “Voice and give voice": dialectics between fiction and history in narratives on the Rwandan genocide w,
International Journal of Francophone Studies, vol. $9, n^{\circ} 1, p$. $93-110$. - Lanzmann, Claude (1995): « The Obscenity of Understanding : An Evening with Claude Lanzmann », in Cathy Caruth (dir.), Trauma:

- Littell, Jonathan (2006) : Les Bienveillantes, Paris, Gallimard.

- Littell, Jonathan et Millet, Richard (2007) : « Conversation à Beyrouth», Le Debar, $n^{\circ}$ 144, 2007, p. 4-24.

- Milkovitch-Rioux, Catherine (2010) : « La guerre d'Algérie, ou la violence en miroir s, in Déborah Lévy-Bertherat et Pierre Schoentjes
(dir.), Já i tué. Violence guerrière et fiction, Genève Droz p 181-193. - Riffaterere, Michael (1995) : « L Le témoignage litérárire », Cahiers de la

Rotman, Patrick (2007) : Lénnemi intime [2002], Paris, Seuil.

- Spiessens, Anneleen (2016): Quand le bourreau prend la parole.

- Venuti, Lawrence (1995): The Translator's Invisibility: A History of
Translation, Londres et New York Routledge

----(1998): The Scandals of Translation: Towards an Ethics of Difference,
Londres, Routledge.

Viart, Dominique et Vercier, Bruno (2008) : La Littérature française au

Vidal, Claudine (2006) : « Un génocide à la machette », in Marc Le Pape, Johanna Siméant et Claudine Vidal (dir)., Crises extrêmes. Face
aux massacres, aux guerres civiles et aux génocides, Paris, La Découverte. p. 21-35.

- Waller, James (2002) : Becoming Evil: How Ordinary People Comm Genocide and Mass Killing, Oxford University Press. Welzer, Harald (2007): : Les exécutueurs. Des hommes normaux aux
meurtriers de masss, traduit de lallemand par Berrard Lortholary, Paris, meurtriers de masse, tradui do 